\title{
Double Contrast-Enhanced
} Ultrasonography in Preoperative T Staging of Gastric Cancer: A Comparison With Endoscopic Ultrasonography

\author{
Liang Wang ${ }^{1}$, Zhe Liu ${ }^{1,2}$, Hongju Kou ${ }^{1}$, Huiliao He ${ }^{2}$, Bo Zheng ${ }^{3}$, Lingling Zhou ${ }^{4}$ and \\ Yan Yang ${ }^{1 *}$
}

${ }^{1}$ Department of Ultrasound, The Second Affiliated Hospital and Yuying Children's Hospital of Wenzhou Medical University, Wenzhou, China, ${ }^{2}$ Wenzhou Institute of Biomaterials and Engineering, Chinese Academy of Sciences, Wenzhou, China, ${ }^{3}$ Department of Gastroenterology, The Second Affiliated Hospital and Yuying Children's Hospital of Wenzhou Medical University, Wenzhou, China, ${ }^{4}$ Department of Pathology, The Second Affiliated Hospital and Yuying Children's Hospital of Wenzhou Medical University, Wenzhou, China

OPEN ACCESS

Edited by:

Cicero Matthew R. Habito,

Massachusetts General Hospital and

Harvard Medical School,

United States

Reviewed by:

Angelo Don II Santos Grasparil, Cardinal MRI Center, Inc., Philippines

Laurence Gluch,

The Strathfield Breast Centre,

Australia

*Correspondence:

Yan Yang

yang25yan1997@163.com

Specialty section:

This article was submitted to Cancer Imaging and Image-directed

Interventions,

a section of the journal

Frontiers in Oncology

Received: 03 May 2018 Accepted: 24 January 2019 Published: 12 February 2019

Citation:

Wang L, Liu Z, Kou H, He H, Zheng B, Zhou L and Yang Y (2019) Double

Contrast-Enhanced Ultrasonography in Preoperative T Staging of Gastric

Cancer: A Comparison With

Endoscopic Ultrasonography.

Front. Oncol. 9:66.

doi: 10.3389/fonc.2019.00066
Objective: To compare the precision of double contrast-enhanced ultrasonography (DCEUS) to endoscopic ultrasonography (EUS) in preoperative $T$ staging of gastric cancers.

Methods: This retrospective study consisted of 158 pathologically confirmed gastric cancer patients. All patients underwent DCEUS (intravenous contrast-enhanced ultrasonography combined with oral contrast-enhanced ultrasonography) and endoscopic ultrasonography (EUS) preoperatively. The histopathological findings of resected specimens were compared with the results of DCEUS and EUS retrospectively.

Results: The accuracy of DCEUS and EUS in evaluating the T staging of gastric cancer were 82.3\% (T1 62.5\%,T2 84.4\%,T3 87.9\%,T4 91.3\%) and 76.6\% (T1 84.4\%,T2 82.2\%,T3 72.4\%,T4 65.2\%), respectively. There were no significant differences between the methods for the overall T staging accuracy $\left(\chi^{2}=1.569, P=0.210\right)$. But EUS was superior to DCEUS for T1 stage $\left(\chi^{2}=3.925, P=0.048\right)$ and DCEUS was superior to EUS for T3 stage $\left(\chi^{2}=4.393, P=0.036\right)$ and T4 stage $\left(\chi^{2}=4.600, P=0.032\right)$.

Conclusion: DCEUS is a convenient and noninvasive method with high precision, which can be used as the primary imaging technique for advanced gastric cancer $T$ staging. In early gastric cancer, we should prefer EUS. Two methods are complementary for assessing tumor invasion depth of gastric cancer.

Keywords: ultrasonography, endoscopic ultrasonography, gastric cancer, surgery, histopathology

\section{INTRODUCTION}

Gastric carcinoma is a highly lethal malignant tumor. It is a serious public health problem in Eastern Asia, Eastern Europe, Central and South America (1). Gastric cancer ranks the fourth among all cancers (nearly 1,000,000 new cases per year) and ranks the third among all cancer deaths worldwide $(2,3)$. Despite recent improvement in diagnosis and therapeutic methods, prognosis of 
gastric cancer remains poor (4). Surgery is still the right choice for the malignancy (5). Accurate preoperative staging to select a reasonable range of surgical and adjuvant therapy program, to avoid over-treatment or inadequate treatment are essential $(6,7)$. The depth of tumor invasion is an important indicator for predicting a patient's prognosis when suffering from gastric carcinoma (8). So, it is important to explore reliable and effective techniques for preoperative $\mathrm{T}$ staging of gastric cancer.

Many modalities, such as barium radiography, gastroendoscopy, CT, and MRI are used to stage gastric tumors $(9,10)$. Nevertheless, until now, no suitable tumor screening method for gastric carcinoma has been suggested by the World Health Organization (11). EUS is regularly utilized to identify and stage gastrointestinal cancers and provides detailed images $(12,13)$. Many researchers have studied the function of EUS in the preoperative staging of gastric cancer, and EUS is regularly contemplated as the primary imaging tool for regional staging of gastric carcinoma as compared with other methods (13-16). However, patient discomfort and risk of cross-infection hamper its application.

We need to identify a noninvasive, simple, economic and reliable approach in modern times. Double contrast-enhanced ultrasonography (DCEUS) has developed as an innovative modality to screen the diseases of gastrointestinal tract such as gastric tumors and rectal neoplasms in China $(17,18)$. SonoVue is an intravenous contrast agent of sulfur hexafluoride microbubbles (19). Combining ultrasonic oral contrast agent (UOCA) and SonoVue in patient examination, it is easy for us to detect gastric carcinoma, giving a precise T-staging. This study reviewed 158 gastric carcinoma cases and compared DCEUS with EUS in surgical outcomes to investigate the importance of DCEUS in the preoperative T-staging of the disease.

\section{METHODS}

This study was carried out in agreement with the Declaration of Helsinki. The protocol was sanctioned by the Research Ethics Committee of the Second Affiliated Hospital of Wenzhou Medical University. All patients gave written informed consent.

\section{Patients}

Between January 2015 and July 2017, 183 consecutive subjects were diagnosed with gastric cancer at our Hospital. The inclusion criteria for this study were: (1)gastric carcinoma as confirmed by endoscopic biopsy; (2)without previous chemotherapy, radiotherapy, immunotherapy or treatment; (3)patients were examined by both DCEUS and EUS a week before surgical resections. The exclusion criteria included: (1) unresectable tumors with widespread metastasis (16 cases); (2)elderly patients with contraindications for surgery (9 cases). A total of 158 patients were included in the final study [52 females, 106 males, average of $59.5 \pm 10.6$ years of age (range 33-80)].

\section{Equipment's and Contrast Agents}

DCEUS examinations were performed with Acuson Seioquoia 512 ultrasound system, equipped with contrast pulse sequencing (CPS) technology; UOCA Xinzhang ${ }^{\circledR}$ (Huqingyutang,
HangZhou, China) was made from a soya derivative; Intravenous contrast agent SonoVue (Bracco, Milan, Italy)-a suspension of sulfur hexafluoride microbubbles.

EUS studies were performed with EndoEcho system (Olympus, Japan): Model for the host EU-M2000; endoscopic ultrasonography for the Olympus GF-UM 2000-ring endoscopic ultrasound scan, the department tip diameter of $12.7 \mathrm{~mm}$, pipe pliers diameter of $2.2 \mathrm{~mm}$, scan range of $360^{\circ}$; Olympus UM-DP12-25R, and UM-DP20-25R ultrasonic micro-probe; ultrasonic probe drive MAJ-935; MH-303 bladders (Japan); sterile degassed water (our hospital).

\section{Examination and Observation DCEUS Examination}

All patients were fasting more than $8 \mathrm{~h}$ and received atropine sulfate $(0.05 \mathrm{mg} / \mathrm{kg})$ intramuscularly $30 \mathrm{~min}$ before examinations to minimize the gastric peristalsis. Firstly, a basic $2 \mathrm{D}$ ultrasound examination was performed by using $4 \mathrm{~V} 1$ probe to identify each gastric lesion. Then the patients ingested UOCA $(500 \mathrm{ml})$ which fills the stomach, and were examined in the supine, and both decubitus positions. The tumors were observed, the sizes of masses were determined, the echoic features and shape of lesions were described. Further steps were performed following a bolus $2.4 \mathrm{ml}$ Sonovue injection. The contrast pulse sequencing (CPS) mode was used when we performed DCEUS. The settings were as follows: acoustic power, -15 to $-21 \mathrm{~dB}$; transmit frequency, 1.5 $\mathrm{MHz}$; frame rate, 17-20. A low $(<0.2)$ mechanical index was used to prevent microbubble disruption. The enhancement patterns of the arterial phase, the venous phase, and the late phase were stored. The storage is up to $5 \mathrm{~min}$. All baseline $2 \mathrm{D}$ and dynamic DCEUS images were recorded on tapes. The images were then assessed by two impartial off-site sonographers. Both of them were blinded to the clinical data, pathology results and other imaging findings of the patients at the time of the analysis.

\section{EUS Examination}

Patients were fasting for more than $8 \mathrm{~h}$. The Olympus GFUM2000 EUS scan-ring were inserted into the level of the duodenum, detailed inspection from the beginning of the duodenum to the esophagus, including duodenum, pylorus, antrum, gastric body, fundus, cardia, esophagus, and the organs around digestive tract, such as the pancreas, part of the liver, spleen, retroperitoneum around the aorta, mediastinum were all observed. The endoscope was advanced beyond the tumor mass. Serial images were obtained when the transducer was pulled back. In order to increase the surface contact and improve the acoustic window, the transducer was surrounded by an inflatable balloon which was filled with deaerated water (20). The sizes of lesions, borders, depths, surrounding organs were all observed. Endoscopic images of the target lesions were subsequently analyzed by two other independent off-site physicians having over 10 years experience. Both of them were unaware of the patients' clinical symptoms, signs, laboratory tests, other imaging results.

The T staging criteria of both DCEUS and EUS are based on the five-story structure of the gastric wall $(21,22)$. T1: tumorinfiltrating limited to the first 1 to 3 layers, that is located in 

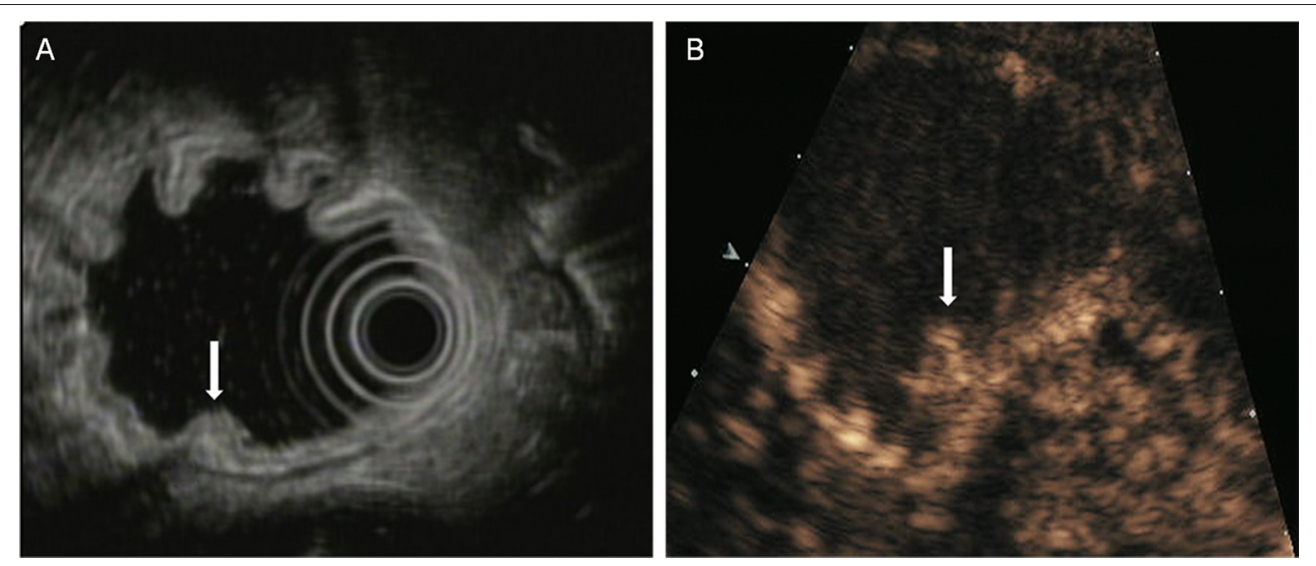

FIGURE 1 | A case with T1 tumor located in the posterior wall of antrum. (A) EUS showed an small elevated lesion (arrow) involving the antral mucosa and submucosa. (B) DCEUS showed the lesion (arrow) invaded into the submucosal layer.
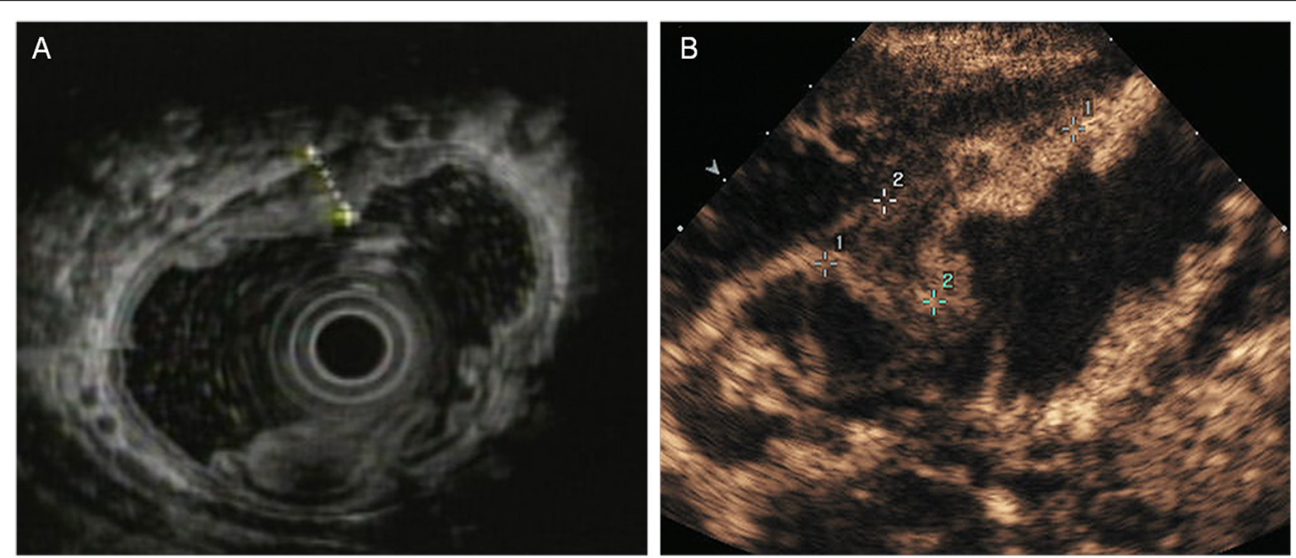

FIGURE 2 | A case with T2 tumor located in the anterior wall of the gastric body. (A) EUS showed an ulcerative lesion involving the muscularis propria (fourth layer). The serosal layer appears intact. (B) DCEUS image showed the lesion invaded into the muscularis propria. The lesion didn't penetrate the serosa.
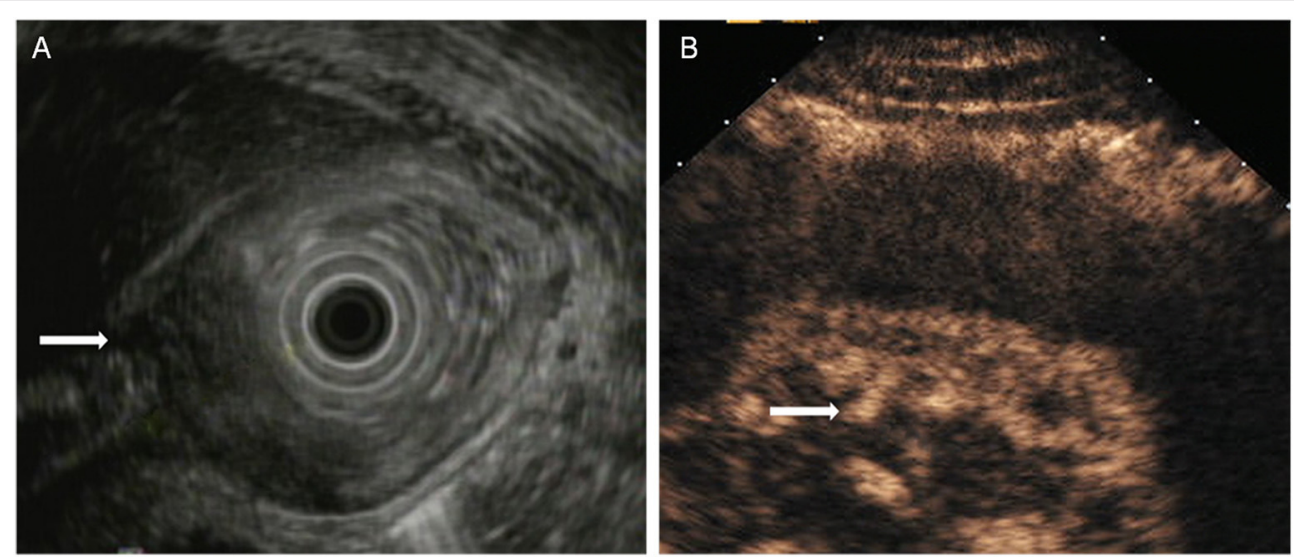

FIGURE 3 | A case with T3 tumor located in the gastric angle. (A) EUS showed an large lesion with a thickness of approximately $3 \mathrm{~cm}$ penetrated the serosal layer. (B) DCEUS showed the lesion penetrated the serosa (arrow) with disappearance of all layers. 

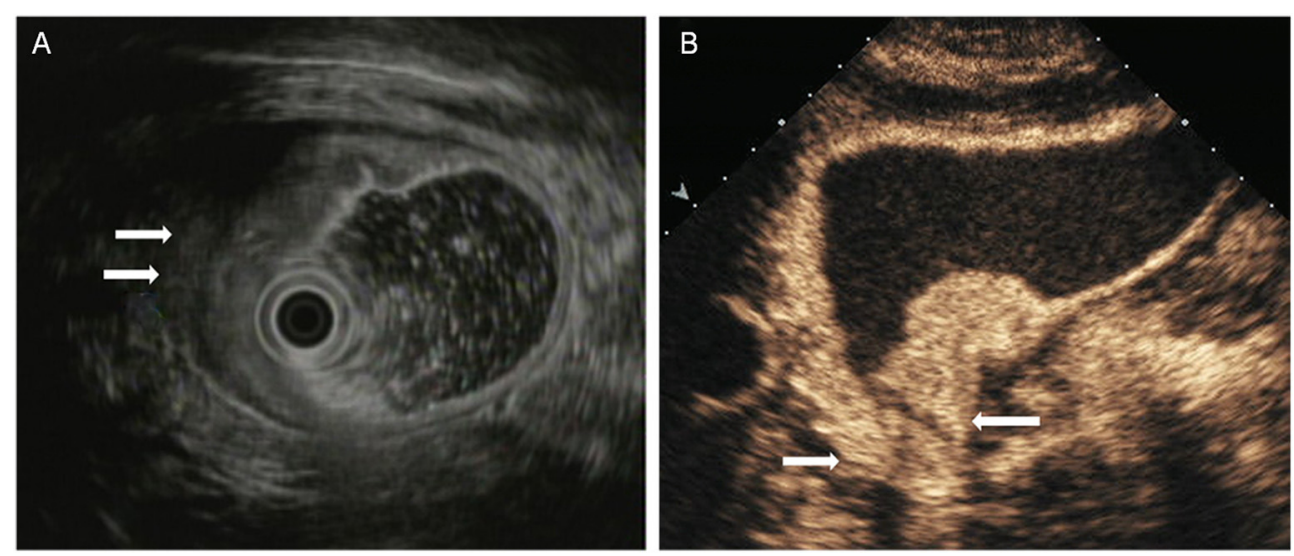

FIGURE 4 | A case with T4 tumor located in the antrum. (A) EUS showed an lesion penetrated the serosal layer and invaded into adjacent tissues (arrow). (B) DCEUS showed the lesion infiltrated to duodenum bulb (arrow).

mucosa or submucosa (Figure 1); T2: tumor invasion to the fourth layer, that is up to the inherent muscle (Figure 2); T3: tumor invasion to the fifth layer, that is, invasion to serosa layer (Figure 3); T4: tumor invasion to the serosa adjacent tissues or organs (Figure 4).

The surgical specimens were transported to the pathology department after operations. The microscopic staging of the resected specimens were performed by the pathologist $(\mathrm{L} \mathrm{Z}$, with 8 years of proficiency), who was unaware of the DCEUS and EUS findings.

\section{Statistical Analysis}

Data analysis was performed by using SPSS version 22.0 software. The diagnostic performance change from DCEUS to EUS was measured by chi-square test. All $P$-values were derived from 2tailed tests, and $P<0.05$ was selected to designate a statistically significant difference.

\section{RESULTS}

All 158 patients undertook surgery. The diameters of resected gastric lesions were in the range of $1.2-11.6 \mathrm{~cm}$ (mean $5.2 \pm$ $1.6 \mathrm{~cm}$ ). Among 158 patients, the tumors were mostly located in the antrum and pylorus region $(n=66)$, followed by the proximal cardia region $(n=50)$.

The histopathological classifications were as follows: well-differentiated adenocarcinoma $=22$ cases, moderately differentiated adenocarcinoma $=33$ cases, poorly differentiated adenocarcinoma $=65$ cases, undifferentiated adenocarcinoma $=13$ cases, signet-ring cell carcinoma $=16$ cases, mucinous adenocarcinoma $=8$ cases, and squamous carcinoma $=1$ case. There were 32 and 126 cases of early and advanced gastric cancer, respectively.

Tumor depth invasion was categorized as follows: $\mathrm{T} 1=32$ cases, $\mathrm{T} 2=45$ cases, $\mathrm{T} 3=58$ cases, and $\mathrm{T} 4$ tumors $=23$ cases. One hundred and thirty cases were correctly staged by DCEUS and 121 cases were correctly staged by EUS, respectively. For DCEUS, the overall $\mathrm{T}$ staging accuracy was $82.3 \%$, with
TABLE 1 | The results of T staging by DCEUS compared with postoperative pathological findings.

\begin{tabular}{lcccccc}
\hline \multicolumn{5}{c}{ DCEUS } & & \\
\cline { 2 - 4 } Pathology & $\mathbf{T}_{\mathbf{1}}$ & $\mathbf{T}_{\mathbf{2}}$ & $\mathbf{T}_{\mathbf{3}}$ & $\mathbf{T}_{\mathbf{4}}$ & Total & Accuracy (\%) \\
\hline $\mathrm{T}_{1}$ & 20 & 12 & & & 32 & 62.5 \\
$\mathrm{~T}_{2}$ & 3 & 38 & 4 & & 45 & 84.4 \\
$\mathrm{~T}_{3}$ & & 4 & 51 & 3 & 58 & 87.9 \\
$\mathrm{~T}_{4}$ & & & 2 & 21 & 23 & 91.3 \\
\hline
\end{tabular}

DCEUS, double contrast-enhanced ultrasonography. The overall accuracy of DCEUS in $T$ staging was $82.3 \%$ (130 of 158 patients).

each stage as follows:T1 staging accuracy $=62.5 \%$, T2 staging accuracy $=84.4 \%$, T3 staging accuracy $=87.9 \%$, and T4 staging accuracy $=91.3 \%$. A total of 19 cases were overstaged (12 patients with T1 overstaged as T2; 4 patients with T2 overstaged as T3; 3 patients with T3 overstaged as T4) and 9 cases were understaged ( 3 patients with T2 understaged as T1; 4 patients with T3 understaged as T2; 2 patients with T4 understaged as T3) (Table 1). For EUS, the overall T staging accuracy was $76.6 \%$, with each stage as follows: $\mathrm{T} 1=84.4 \%, \mathrm{~T} 2=82.2 \%, \mathrm{~T} 3=$ $72.4 \%$, and $\mathrm{T} 4=65.2 \%$. A total of 17 cases were overstaged ( 5 patients with T1 overstaged as T2; 6 patients with T2 overstaged as T3; 6 patients with T3 overstaged as T4) and 20 cases were understaged ( 2 patients with $\mathrm{T} 2$ understaged as T1; 10 patients with T3 understaged as T2; 8 patients with T4 understaged as T3) (Table 2).

Statistically, there was no significant difference between two methods for the overall $\mathrm{T}$ staging accuracy $\left(\chi^{2}=1.569, P=\right.$ $0.210)$. But EUS was superior to DCEUS for T1 stage $\left(\chi^{2}=3.925\right.$, $P=0.048)$; DCEUS was superior to EUS for T3 stage $\left(\chi^{2}=4.393\right.$, $P=0.036)$ and T4 stage $\left(\chi^{2}=4.600, P=0.032\right)$ (Table 3$)$.

\section{DISCUSSION}

Gastric cancer is a common malignancy of the digestive tract. Its treatment and survival rates are associated with the early 
TABLE 2 | The results of T staging by EUS compared with postoperative pathological findings.

\begin{tabular}{lcccccc}
\hline \multicolumn{5}{c}{ EUS } \\
\cline { 2 - 5 } Pathology & $\mathbf{T}_{\mathbf{1}}$ & $\mathbf{T}_{\mathbf{2}}$ & $\mathbf{T}_{\mathbf{3}}$ & $\mathbf{T}_{\mathbf{4}}$ & Total & Accuracy (\%) \\
\hline $\mathrm{T}_{1}$ & 27 & 5 & & & 32 & 84.4 \\
$\mathrm{~T}_{2}$ & 2 & 37 & 6 & & 45 & 82.2 \\
$\mathrm{~T}_{3}$ & & 10 & 42 & 6 & 58 & 72.4 \\
$\mathrm{~T}_{4}$ & & & 8 & 15 & 23 & 65.2 \\
\hline
\end{tabular}

EUS, endoscopic ultrasonography. The overall accuracy of EUS in T staging was $76.6 \%$ (121 of 158 patients).

TABLE 3 | Comparison of the two methods in T staging of gastric cancer.

\begin{tabular}{lcccc}
\hline T stage & DCEUS (\%) & EUS (\%) & $\chi^{2}$ & $P$ \\
\hline $\mathrm{T}_{\text {sum }}$ & 82.3 & 76.6 & 1.569 & 0.210 \\
$\mathrm{~T}_{1}$ & 62.5 & 84.4 & 3.925 & $0.048^{*}$ \\
$\mathrm{~T}_{2}$ & 84.4 & 82.2 & 0.080 & 0.777 \\
$\mathrm{~T}_{3}$ & 87.9 & 72.4 & 4.393 & $0.036^{\star}$ \\
$\mathrm{T}_{4}$ & 91.3 & 65.2 & 4.600 & $0.032^{\star}$ \\
${ }^{*} P<0.05$. & & & &
\end{tabular}

diagnosis and accurate clinical staging for a reasonable range of surgery.

Surgical resection of gastric cancer remains the only available treatment and depends on the stage of the disease at presentation. Some early gastric cancer (T1) may be treated with endoscopic submucosal dissection (ESD) and endoscopic mucosal resection (EMR). Lymph node dissection is required for advanced gastric cancer (T2 stage and above). For primary tumors or metastases that directly invade adjacent organs, the affected organs should be removed jointly. And for advanced gastric cancer, adjuvant and neoadjuvant chemotherapy, either alone or in combination with radiation therapy, have been shown to improve survival rates (23). Therefore, accurate preoperative $\mathrm{T}$ stage is very important for planning the optimal surgical procedure.

Reliable preoperative $\mathrm{T}$ staging methods that are consistent with the pathological specimens are necessary prior to developing a treatment plan. However, each of the currently used methods have limitations and no single staging method is acknowledged as the method of choice. Consequently, National Comprehensive Cancer Network practice guidelines for gastric carcinoma do not suggest explicit methods and recommend the use of various modalities supplementary as staging technique (24).

EUS combines the advantages of endoscopy and ultrasound. It can not only display the location, shape, size, internal echo of the tumor, but also provides detailed images of the malignancy infiltrating depth. For the T staging of gastric carcinoma, EUS has been identified as the preferred diagnostic method $(25,26)$. There are however, several opposing statements about the reliability of EUS in determining the $\mathrm{T}$ stage since described values for EUS diagnostic accuracy in overall $\mathrm{T}$ staging ranged from 42.6 to $87.7 \%(26-28)$. In this research, the general accuracy of
EUS was 76.6\%; Among the different stages, the accuracy was $84.4 \%$ for T1, $82.2 \%$ for T2, $72.4 \%$ for T3, and $65.2 \%$ for T4. A total of $17(10.8 \%)$ cases were overstaged and $20(12.7 \%)$ cases were understaged.

Traditional transabdominal ultrasound is unable to adequately stage the depth of gastric tumors, due to variations of wall thickening frequently being too delicate to visualize (29). Double contrast-enhanced ultrasonography is a relatively new method. It is a transabdominal ultrasound method utilizing both intraluminal and intravenous contrast to improve ultrasonographic visualization. The use of UOCA can distend the gastric lumen and displace the air in the stomach, therefore helping to display mucosal lesions (30). The use of intravenous contrast can demonstrate blood perfusion of the tumors and enhance visualization of lesions through the arterial phase to identify the invasion depth. Thus, DCEUS can display morphologic appearances and perfusion characteristics of both normal and abnormal structures (31). UOCA can discharge intragastric air and form a homogeneous distribution of ultrasonic transmission surface. This may lead to a reduction in ultrasonic artifacts and provides a good acoustic window, to increase the detection rate of the gastric lesions. But it is difficult for oral contrast enhancd ultrasound to differentiate tumor tissues from inflammation and fibrosis due to the small acoustic impedance difference and the limitation of resolution (32). And this is the most common reason for overestimation or underestimation while using single oral contrast enhancd ultrasound (20, 33, 34). Angiogenesis and infiltration are essential for the invasive growth of tumors (35). Single oral contrast enhancd ultrasound cannot show the microvascular perfusion of the tumors. As a blood pool agent, SonoVue can, through blood circulation, enter the capillary of gastric lesions. It can create strong echoes over a range of frequencies routinely utilized in medical ultrasound examinations (19). Combining UOCA and SonoVue is useful to stage gastric malignancy before surgery. In this study, the general accuracy of DCEUS for $\mathrm{T}$ staging was $82.3 \%$; Among the different stages, the accuracy was $62.5 \%$ for $\mathrm{T} 1,84.4 \%$ for $\mathrm{T} 2,87.9 \%$ for $\mathrm{T} 3$, and $91.3 \%$ for $\mathrm{T} 4$. Tumors enhanced in the arterial phase and agents washed out in the venous phase, which made the borders of lesions clearer. Therefore, the tumor's contour and invasive depth can be easily identified. Overestimation and underestimation also existed in $\mathrm{T}$ staging using DCEUS. A total of 19 (12.0\%) cases were overstaged and $9(5.7 \%)$ cases were understaged.

In this study, the overall accuracy of DCEUS was similar to that of EUS in determining the T stage. But EUS was superior to DCEUS for T1 stage and DCEUS was superior to EUS for T3 and T4 stage. EUS is used to visualize the lesion surface, portraying interference of abdominal fat and gas in the stomach. Thus, EUS is more accurate in identifying stages early in well-differentiated carcinomas (Figure 5) (36). The accuracy of EUS in late stage tumors was reduced. A lesion-by-lesion analysis revealed that overestimation of EUS mostly present in the cases of malignancy with ulcerative type. Using EUS it is difficult to differentiate fibrosis and inflammation from tumors, which is a common cause of misreading of depth in ulcerative malignancy. Moreover, we found underestimation of invasion 

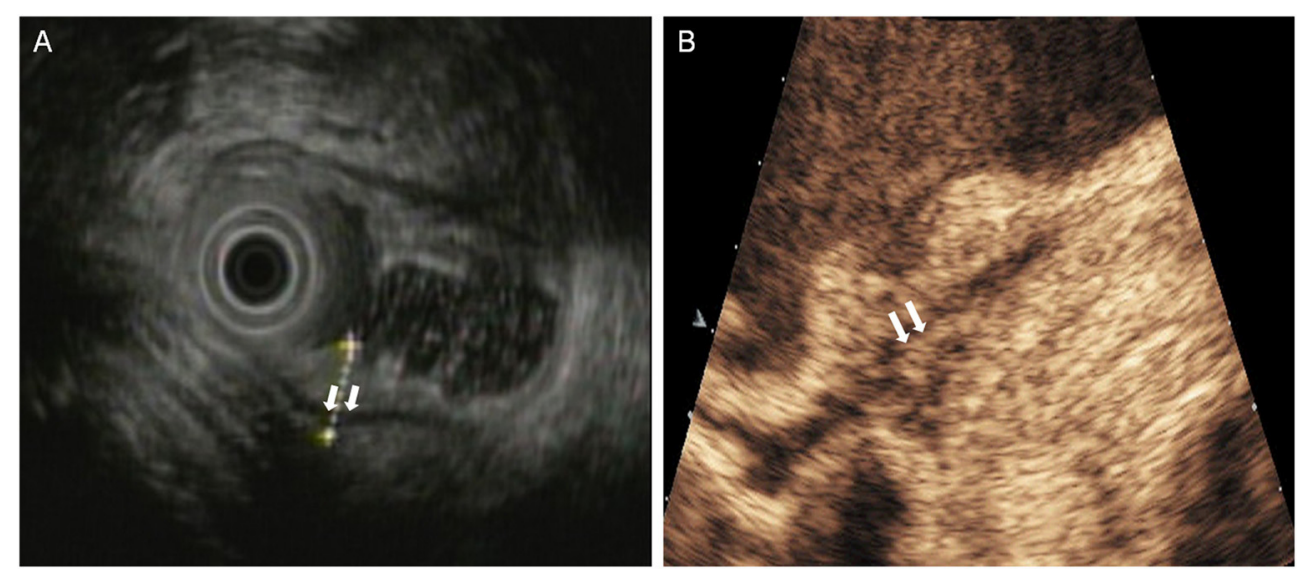

FIGURE 5 | A case with T1 tumor was overstaged by DCEUS but was accurately staged by EUS. (A) EUS showed a lesion invaded into the submucosa, and the muscularis propria was intact (arrow), but (B) DCEUS showed the lesion invaded into the muscularis propria (arrow) in error and overstaged it as T2 tumor.
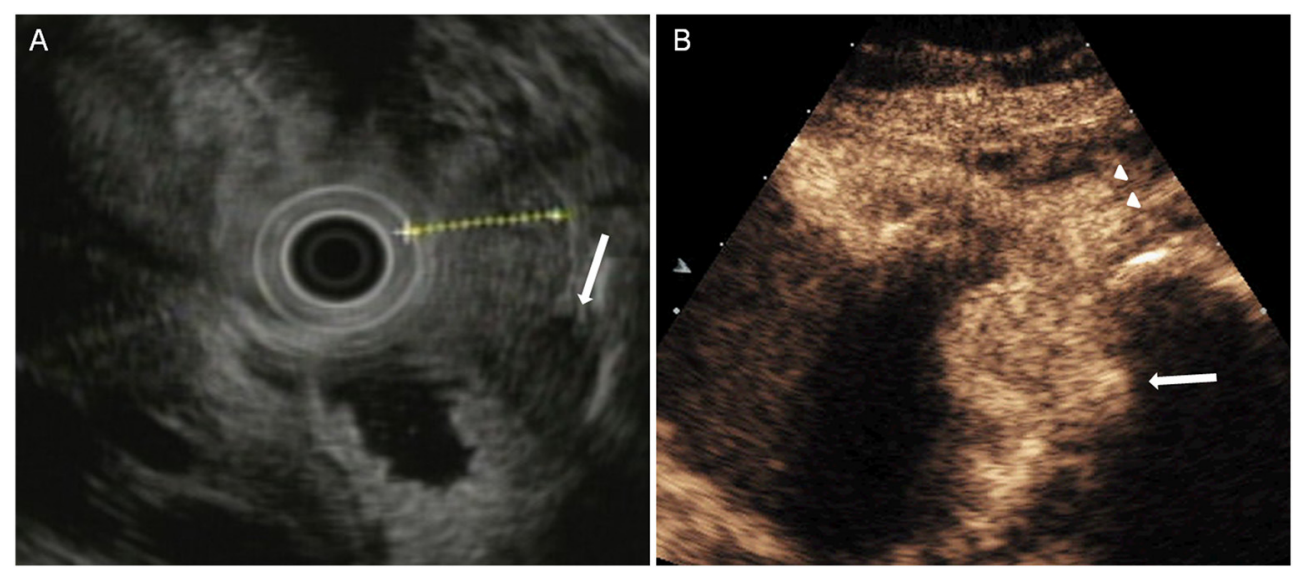

FIGURE 6 | A case with T4 tumor was understaged by EUS but was accurately staged by DCEUS. (A) EUS showed an lesion penetrated the serosa (arrow) and understaged it as T3 tumor. (B) DCEUS showed the lesion not only penetrated the serosal layer (arrow) but also infiltrated to adjacent tissues (triangle).

depth mostly present in the cases of tumors with diameters $>5 \mathrm{~cm}$. This maybe due to the tumors being too large and the frequency of EUS being relatively high, it was hard for EUS to display the whole lesions or to show the views of the maximum depths of infiltration (Figure 6). Contrariwise, DCEUS is better for determining the stage of advanced gastric cancer due to the rich blood supply. The advantage of DCEUS is its high contrast resolution, which can be used to distinguish tumors from normal tissue. Hence, it is sensitive to lesion detection, characterization, and staging. What's more, it can show the relationship of the lesion's vasculature and the gastric wall, in addition to their contours. The lack of vascularity in early gastric cancer leads to its low accuracy. In addition, other factors may also influence its accuracy, such as unsatisfied filling of gastric cavity, artifacts caused by gastric peristalsis.

In our opinion, DCEUS is a viable supplement to the preoperative work-up of biopsy-proven gastric cancer in the preoperative staging of the disease. The findings of DCEUS can be valuable and additive for the suitable treatment plans for gastric cancer patients, in particular for the elder population which invasive EUS carries risks. Furthermore, the cost of doing both EUS and DCEUS is not considerable in our hospital, and the additional information makes the cost of performing both tests worthwhile.

This study was a retrospective study, only enrolling patients referred to our hospital for surgery. This represent a bias issue that may affect accurate evaluation. A prospective study should be performed to avoid the bias in future research.

In conclusion, as a convenient and noninvasive technique with high accuracy, DCEUS can be used as the primary imaging modality for the $\mathrm{T}$ staging of advanced gastric cancer. In early gastric cancer, we should prefer to EUS. Two methods are complementary for assessing tumor invasion depth of gastric cancer. 


\section{AUTHOR CONTRIBUTIONS}

LW and YY: designed this study. LW, HK, and BZ: acquired the data. $\mathrm{ZL}, \mathrm{HH}$, and $\mathrm{LZ}$ : interpreted the data. LW: wrote and edited the manuscript. All authors reviewed the manuscript.

\section{REFERENCES}

1. Zhou J, Ma X, Bi F, Liu M. Clinical significance of circulating tumor cells in gastric cancer patients. Oncotarget (2017) 8:25713-20. doi: 10.18632/oncotarget.14879

2. Ferlay J, Soerjomataram I, Dikshit R, Eser S, Mathers C, Rebelo M, et al. Cancer incidence and mortality worldwide: sources, methods and major patterns in GLOBOCAN 2012. Int J Cancer (2015) 136:E359-86. doi: 10.1002/ijc.29210

3. Torre LA, Bray F, Siegel RL, Ferlay J, Lortet-Tieulent J, Jemal A. Global cancer statistics, 2012. CA Cancer J Clin. (2015) 65:87-108. doi: 10.3322/caac.21262

4. Correia M, Machado JC, Ristimäki A. Basic aspects of gastric cancer. Helicobacter (2009) 14(Suppl. 1):36-40. doi: $10.1111 / j .1523-5378.2009 .00696 . x$

5. Smyth EC, Verheij M, Allum W, Cunningham D, Cervantes A, Arnold D. Gastric cancer: ESMO clinical practice guidelines for diagnosis, treatment and follow-up. Ann Oncol. (2016) 27(Suppl. 5):v38-49. doi: 10.1093/annonc/mdw350

6. Kunisaki C, Shimada H, Nomura M, Matsuda G, Otsuka Y, Ono $\mathrm{H}$, et al. Distribution of lymph node metastasis in gastric carcinoma. Hepatogastroenterology (2006) 53:468-72.

7. Lim JS, Yun MJ, Kim MJ, Hyung WJ, Park MS, Choi JY, et al. CT and PET in stomach cancer: preoperative staging and monitoring of response to therapy. Radiographics (2006) 26:143-56. doi: 10.1148/rg.261 055078

8. Spolverato G, Ejaz A, Kim Y, Squires MH, Poultsides GA, Fields RC, et al. Endoscopic ultrasound in the preoperative staging of gastric cancer: a multi-institutional study of the US gastric cancer collaborative. J Am Coll Surg. (2015) 220:48-56. doi: 10.1016/j.jamcollsurg.2014. 06.023

9. Guo J, Liu Z, Sun S, Wang S, Ge N, Liu X, et al. Endosonographyassisted diagnosis and therapy of gastrointestinal submucosal tumors. Endosc Ultrasound (2013) 2:125-33. doi: 10.7178/eus.06.003

10. Bhutani MS. Endoscopic ultrasound comes of age: mature, established, creative and here to stay! Endosc Ultrasound (2014) 3:143-51. doi: 10.4103/2303-9027.138782

11. Liu Z, Guo J, Wang S, Zhao Y, Li J, Ren W, et al. Evaluation of transabdominal ultrasound after oral administration of an echoic cellulose-based gastric ultrasound contrast agent for gastric cancer. BMC Cancer (2015) 15:932. doi: 10.1186/s12885-015-1943-0

12. Han C, Lin R, Liu J, Hou X, Qian W, Ding Z. Endoscopic ultrasonographyguided biopsy for differentiation of benign and malignant pelvic lesions: a systematic review and meta-analysis. Dig Dis Sci. (2015) 60:3771-81. doi: $10.1007 /$ s10620-015-3831-5

13. Han $\mathrm{C}$, Lin $\mathrm{R}$, Yu J, Zhang Q, Zhang $\mathrm{Y}$, Liu J, et al. A case report of esophageal bronchogenic cyst and review of the literature with an emphasis on endoscopic ultrasonography appearance. Medicine (2016) 95:e3111. doi: 10.1097/MD.00000000000 03111

14. Mehmedović A, Mesihović R, Saray A, Vanis N. Gastric cancer staging: EUS and CT. Med Arch. (2014) 68:34-6. doi: 10.5455/medarh.2014.68.34-36

15. $\mathrm{Wu} \mathrm{CX}, \mathrm{Zhu} \mathrm{ZH}$. Diagnosis and evaluation of gastric cancer by positron emission tomography. World J Gastroenterol. (2014) 20:4574-85. doi: 10.3748 /wjg.v20.i16.4574

16. Mocellin S, Pasquali S. Diagnostic accuracy of endoscopic ultrasonography (EUS) for the preoperative locoregional staging of primary gastric cancer. Cochrane Database Syst

\section{ACKNOWLEDGMENTS}

We would like to thank Dr. Mingdong Lu for his help in finishing this study. And We would like to thank our colleagues in the Department of Gastroenterology and the Department of Pathology for their cooperations.

Rev. (2015) 2:Cd009944. doi: 10.1002/14651858.CD009
944.pub2

17. $\mathrm{Li} \mathrm{T}$, Lu $\mathrm{M}$, Song $\mathrm{J}$, Wu $\mathrm{P}$, Cheng $\mathrm{X}$, Zhang $\mathrm{Z}$. Improvement to ultrasonographical differential diagnosis of gastric lesions: the value of contrast enhanced sonography with gastric distention. PLoS ONE (2017) 12:e0182332. doi: 10.1371/journal.pone.01 82332

18. Lu M, Yan B, Song J, Ping W, Yue LX, Song B. Double-contrastenhanced sonography for diagnosis of rectal lesions with pathologic correlation. J Ultrasound Med. (2014) 33:575-83. doi: 10.7863/ultra.3 3.4 .575

19. Schneider M. SonoVue, a new ultrasound contrast agent. Eur Radiol. (1999) 9:S347-8. doi: 10.1007/PL00014071

20. Ganpathi IS, So JB-Y, Ho K-Y. Endoscopic ultrasonography for gastric cancer: does it influence treatment? Surg Endosc. (2006) 20:559-62. doi: 10.1007/s00464-005-0309-0

21. Liao SR, Dai Y, Huo L, Yan K, Zhang L, Zhang $H$, et al. Transabdominal ultrasonography in preoperative staging of gastric cancer. World J Gastroenterol. (2004) 10:3399-404. doi: 10.3748/wjg.v10.i 23.3399

22. Rubaltelli K, Khadivi Y, Tregnaghi A, Stramare R, Ferro F, Borsato $\mathrm{S}$, et al. Evaluation of lymph node perfusion using continuous mode harmonic ultrasonography with a second generation contrast agent. J Ultrasound Med. (2004) 23:829-36. doi: 10.7863/jum.2004.2 3.6.829

23. Cunningham D, Allum WH, Stenning SP, Thompson JN, Van de Velde CJ, Nicolson $\mathrm{M}$, et al. Perioperative chemotherapy versus surgery alone for resectable gastroesophageal cancer. N Engl J Med. (2006) 355:11-20. doi: 10.1056/NEJMoa055531

24. Ajani JA, Barthel JS, Bekaii-Saab T, Bentrem DJ, D'Amico TA, Das P, et al. Gastric cancer. J Natl Compr Canc Netw. (2010) 8:378-409.

25. Lee YT, Ng EK, Hung LC, Chung SC, Ching JY, Chan WY, et al. Accuracy of endoscopic ultrasonography in diagnosing ascites and predicting peritoneal metastases in gastric cancer patients. Gut (2005) 54:1541-5. doi: 10.1136/gut.2004.055772

26. Cardoso R, Coburn N, Seevaratnam R, Sutradhar R, Lourenco LG, Mahar A, et al. A systematic review and meta-analysis of the utility of EUS for preoperative staging for gastric cancer. Gastric Cancer (2012) 15(Suppl. 1):S19-26. doi: 10.1007/s10120-0110115-4

27. Meyer L, Meyer F, Schmidt U, Gastinger I, Lippert H. Endoscopic ultrasonography (EUS) in preoperative staging of gastric cancer-demand and reality. Pol Przegl Chir. (2012) 84:152-7. doi: 10.2478/v10035-0120024-1

28. Yamamoto S, Nishida T, Kato M, Inoue T, Hayashi Y, Kondo J, et al. Evaluation of endoscopic ultrasound image quality is necessary in endosonographic assessment of early gastric cancer invasion depth. Gastroenterol Res Pract. (2012) 2012:194530. doi: 10.1155/2012/194530

29. Neumaier CE, Cittadini G, Grasso A, Dahmane M. Role of ultrasonography in the staging of gastrointestinal neoplasms. Semin Surg Oncol. (2001) 20:86-90. doi: 10.1002/ssu.1022

30. Barr RG. Ultrasound contrast. Oral and i.v. agents amplify the image. Diagn Imaging (San Franc) (1999) 21:56-61.

31. Pan M, Huang P, Li S, Chen J, Wei S, Zhang Y. Double contrast-enhanced ultrasonography in preoperative borrmann classification of advanced gastric carcinoma:comparison with histopathology. Sci Rep. (2013) 3:3338. doi: $10.1038 /$ srep03338 
32. Afonso R, Franceschi D, Parra J, Livingstone A, Lima M, HamiltonNelson K, et al. Endoscopic ultrasound restaging after neoadjuvant chemotherapy in esophageal cancer. Am J Gastroenterol. (2006) 10:1216-21. doi: 10.1111/j.1572-0241.2006.00692.x

33. Yanai H, Tada M, Karita M, Okita K. Diagnostic utility of 20-megahertz linear endoscopic ultrasonography in early gastric cancer. Gastrointest Endosc. (1996) 44:29-33.

34. Lightdale CJ. Endoscopic ultrasonography in the diagnosis, staging and follow-up of esophageal and gastric cancer. Endoscopy (1992) 24:297-303. doi: $10.1055 /$ s-2007-1010487

35. Folkman, J. Tumor angiogenesis: therapeutic implications. $N$ Engl J Med. (1971) 285:1182-6. doi: 10.1056/NEJM197111182852108

36. Kanamori A, Hirooka Y, Itoh A, Hashimoto S, Kawashima H, Hara K, et al. Usefulness of contrast-enhanced endoscopic ultrasonography in the differentiation between malignant and benign lymphadenopathy. Am J Gastroenterol. (2006) 101:45-51. doi: 10.1111/j.1572-0241.2006.00394.x

Conflict of Interest Statement: The authors declare that the research was conducted in the absence of any commercial or financial relationships that could be construed as a potential conflict of interest.

Copyright (C) 2019 Wang, Liu, Kou, He, Zheng, Zhou and Yang. This is an open-access article distributed under the terms of the Creative Commons Attribution License (CC $B Y)$. The use, distribution or reproduction in other forums is permitted, provided the original author(s) and the copyright owner(s) are credited and that the original publication in this journal is cited, in accordance with accepted academic practice. No use, distribution or reproduction is permitted which does not comply with these terms. 